\title{
A study on the effects of the hydroalcholic extract of the aerial parts of Alhagi camelorum on prolactin and pituitary-gonadal activity in rats with hypercholesterolemia
}

\author{
Ali Zarei ${ }^{1}$, Saeed Changizi Ashtiyani ${ }^{2}$, Gholam Hassan Vaezi ${ }^{3}$ \\ ${ }^{1}$ Young Researchers Club, Abadeh Branch, Islamic Azad University, Abadeh, Iran; \\ ${ }^{2}$ Department of Physiology, Arak University of Medical Sciences, Arak, Iran; \\ ${ }^{3}$ Department of biology, Islamic Azad University, Damghan Branch, Semnan, Iran.
}

\begin{abstract}
Summary Background: Although endocrine disorders are not a common cause of infertility, in some cases, testing thyroid function, and hypothalamus - pituitary - gonadal axis can determine the cause of infertility. We aimed to investigate the effect of the aerial parts of Alhagi camelorum extract on prolactin, cortisol and pituitary - gonadal axis activities in rats with hypercholesterolemia.

Materials and methods: In this study, 35 male wistar rats in 5 groups $(n=7)$ were assigned as: control group with normal diet, the sham group with fat diet and three experimental groups of hypercholesterolaemic animals which received Alhagi camelorum extract at a minimum dose of $100 \mathrm{mg} / \mathrm{kg}$, average dose of $200 \mathrm{ml} / \mathrm{kg}$ and maximum dose of $300 \mathrm{mg} / \mathrm{kg}$ over a period of 21 days. At the end of the period, blood samples were collected from all groups and blood factors were then measured and analyzed.

Results: In the sham group compared to the control, cholesterol levels increased and FSH levels decreased, whereas cholesterol levels reduced in the experimental groups. Alhagi camelorum extract also reduced testosterone level and increased prolactin and gonadotropins. Conclusion: Alhagi camelorum extract at low and average doses reduced cortisol, testosterone and cholesterol and increased gonadotropins. So it can cause reproductive disorders in male rats. The extract at maximum dose can increase cortisol and prolactin. As these two hormones work together to produce milk, this plant can help to boost breastfeeding.
\end{abstract}

KEY WORDS: Alhagi camelorum; Prolactin; Testosterone; Cholesterol; Gonadotropin.

Submitted 8 April 2014; Accepted 30 January 2014

\section{INTRODUCTION}

Today, with advances in science and technology, it is recognized that infertility is not just a problem for women. Male factors are also involved. Infertility is defined as a

No conflict of interest declared. state in which no pregnancy occurs after a year of sex activity without using birth control methods. When discussing infertility, people generally believe that most problems are related to women. In fact, nearly $30 \%$ of infertility problems are related to men and $20 \%$ are common problems between women and men. So, $50 \%$ of men are involved in problems related to infertility. However, this rate is different in different countries and in different studies. Obesity is associated with various hormonal changes that can be responsible for changes in sperm motility and abnormal sexual function. Evidence suggests that in obese men, more androgen changes into estrogen in fat tissue and serum testosterone level decreases. However, by increasing the negative feedback of estrogen on pituitary gland, gonadotropin levels decrease. Pulse obesity also affects GnRH-FSH-LH which may affect sex hormones secretion and sperm maturation by disrupting Leydig and Sertoli cells $(1,2)$. Endocrine disorders are not among the common causes of infertility; however, in some cases, the cause of infertility can be determined by testing thyroid, gonadotropins, prolactin and testosterone. The level of FSH rises with germinal cell aplasia and the level of testosterone in men with hypogonadotropic hypogonadism decreases (1). Leydig cells are the main source of testosterone and have receptors for prolactin which at normal levels increases testosterone secretion. These studies suggest a synergy between prolactin, LH and testosterone. However, high levels of prolactin reduces testosterone and leads to frigidity $(1,2)$. Prolactin is regulated by dopamine and some other factors such as TRH. Dopamine is a neurotransmitter that has an inhibitory effect on the hypothalamic-pituitary-gonadal axis (2). TRH, secreted from the hypothalamus gland, stimulates prolactin secretion.

Cholesterol is the precursor of steroid hormones and cholesterol changes into pregnenolone by $\mathrm{P}_{450}$ in mitochondria $(1,3,4)$. On the other hand, increased level of blood cholesterol is associated with coronary artery disease, fat liver and infertility. Excessive fat causes the male hormone of testosterone to be converted to estrogen which reduces the production of sex cells. 
Cholesterol levels can be lowered by diet or drugs $(1,4)$. Many plants and compounds can be effective in reducing cholesterol. Alhagi camelorum is one of the plants in traditional medicine which is used to treat metabolic, gastrointestinal and liver diseases, rheumatic disorder, migraines and warts. Laboratory studies indicate that Alhagi camelorum extract reduces body temperature and heart rate. The extract also inhibits the action of acetylcholine to relax the muscles and is helpful in opening the urinary tract and disposal of kidney stones (5).

This plant, commonly called camel thorn (Figure 1), with the scientific name of Alhagi maurorum belongs to the plant family of leguminosae (papilionaceous). The family has about 550 genera and more than 13000 species (6). Other chemical researches on this plant indicate that it contains sterols and fatty acids $(7,8)$, flavonoids $(9$, 10 ), coumarins (8), alkaloids $(6,8)$, and vitamins. About 12 types of flavonoids have been isolated from this plant (1). Studies have shown that flavonoids have anti- androgenic and antifertility effects on the reproductive system of dogs $(12,13)$.

Studies on the bioactive compounds which have the potential to inhibit or stop cancer cells can pave the way to discover more effective drugs (14). Nowadays, people are increasingly using fruits and vegetables due to their protective effects against illnesses like cancer, cardiovascular and liver diseases $(15,16)$. This is due to the antioxidant compounds present in plants, including vitamins B and C, carotenoids, lycopene and flavonoids, which prevent the damages caused by free radicals $(17,19)$.

As infertility and lipid disorders are increasing and most of the anti-fat drugs and contraceptives available in the pharmaceutical market of Iran and the world have multiple side effects, and also thanks to the increasing tendency towards herbal remedies because of their fewer side effects, any study on medicinal plants is of great value (4). With this in mind, the present study aimed to investigate the effects of the extract of the aerial parts of Alhagi camelorum on prolactin and pituitary-gonadal axis activities in rats with hypercholesterolemia.

Figure 1.

The aerial parts of camel thorn (Alhagi Camelorum).

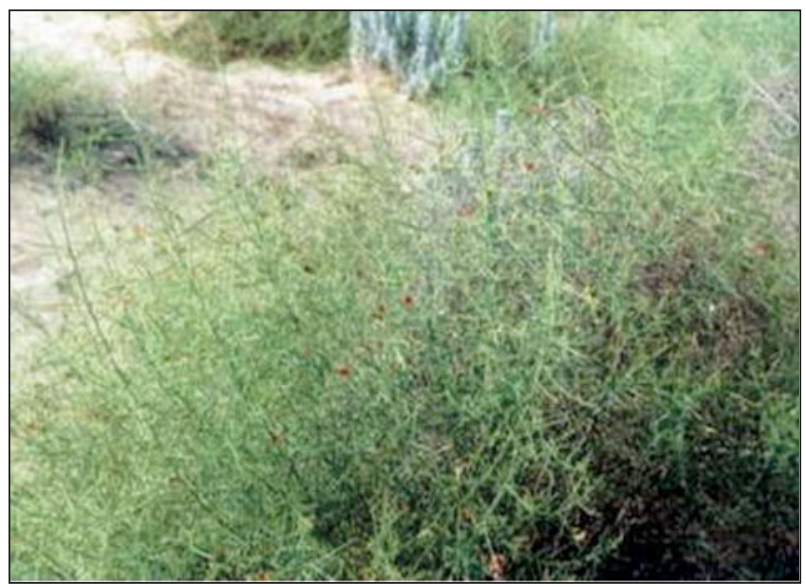

\section{Methods}

This is an experimental study .All animals were taken from Razi Institute in Fars Province and were kept in standard conditions of temperature and light. This study is based on observing all moral codes of working with laboratory animals established by the Ministry of Health and Medical Training (Iran). Before the research, All the animal were weighed to be within a certain weight range. Initially, 35 male wistar rats with the average weight of 5 \pm 170 g were randomly divided into 5 groups $(n=7)$ as follows: controls - the animals in this group did not receive any drug or solvent during the experiment and their diet was normal. Sham group consisted of hypercholesterolemic rats which received $0.2 \mathrm{ml}$ of solvent (normal saline) for 21 days as gavage; (2\% cholesterol was added to their food to make them hypercholesterolemic); experimental group 1, hypercholesterolaemic rats which received a minimum dose of $100 \mathrm{mg} / \mathrm{kg}$ of Alhagi camelorum extract for 21 days as gavage; experimental group 2: hypercholesterolemic rats that were gavaged for 21 days with an average dose of $200 \mathrm{mg} / \mathrm{kg}$ of the extract; and experimental group 3 were hypercholesterolemic rats receiving maximum dose of $300 \mathrm{mg} / \mathrm{kg}$ of the extract for the same period as gavage feeding.

\section{Preparation method for high Cholesterol fOOd}

To obtain a 2\% high-cholesterol diet, 20 grams of Merck pure cholesterol powder (Fluke Chemika) was solved in 5 $\mathrm{ml}$ of olive oil and the solution was well mixed with a kilogram of rat diet. To avoid deterioration of the food it was kept in the refrigerator for only two days $(20,21)$.

\section{EXTRACTION}

Alhagi camelorum plants were collected from the suburb of Abadeh (Fars Province/Iran) and were identified and confirmed by the PNU (Payame Noor University) Department of Botany (herbarium code was 002/040/073). To prepare the Alhagi camelorum alcoholic extract, after providing the aerial parts and removing impurities, 800 grams of the collected plant samples were crushed and mixed with ethyl alcohol $98 \%$ by the ratio of 1 to 5 .

The content obtained was kept in a package for 48 hours in vitro and it was carefully filtered by passing it through different small and big filters. Then it was placed in a water bath to concentrate. Finally, different concentrations of the obtained extract (about $15 \mathrm{~g}$ per $100 \mathrm{~g}$ of crushed plant) was prepared by adding different amounts of normal saline.

During the experimental period all experimental groups were fed with high fat diet. During the test period (21 days) the animals were daily injected at $9 \mathrm{am}$. After completing this course and in order to measure plasma biochemical factors of the animals, they were mildly anesthetized with ether and their blood was collected and then centrifuged at $3000 \mathrm{rpm}$. The serum was separated and transferred to the laboratory for measurement of factors. To measure cholesterol, prolactin, testosterone, cortisol, and gonadotropin RIA (RIA), Pars Azmoon kits and RIA 1000 machine (made in USA) were used. For statistical analysis the mean obtained (mean \pm SEM), one way 
ANOVA test and Tukey and Duncan tests were used. All statistical analyses were done using SPSS software version $17(\mathrm{P}<0.05)$.

\section{RESULTS}

As shown in Table 1 the amount of cholesterol in the sham group increased significantly compared to the control group and in the group receiving the minimum dose of the extract the cholesterol level significantly decreased compared to the sham group $(\mathrm{P}=0.02)$. The differences between experimental groups are not significant.

In the case of FSH, mean value in the sham group shows a significant decrease than in the control group. And the group receiving an average dose of Alhagi camelorum extract shows a significant increase compared to the sham group and the groups receiving the minimum and maximum doses of the extract $(P=0.001)$.

LH level in the sham group did not show significant changes than in the control group. However, in groups with minimum and average doses there is a significant increase compared to both the sham group and the group receiving the highest dose of the extract $(\mathrm{P}=$ 0.01 ).

In the case of testosterone in the sham group no significant change was seen if weighed against the control group, however, the amount of it in groups receiving minimum and average doses a significant decrease was seen compared to the sham group $(\mathrm{P}=0.02)$. None of the changes in experimental groups were significant.

Prolactin level increased in the sham group compared to the control group, but these changes were not significant. Its level in the group receiving the maximum dose showed a significant increase as compared to the sham group and the groups which received minimum or average doses of the extract $(P=0.007)$. However, no signif-

\section{Table 1.}

Effects of different doses of the extract of the aerial parts of Alhagi Camelorum (AC) on cholesterol, cortisol, testosterone, prolactin and gonadotropin.

\begin{tabular}{|c|c|c|c|c|c|}
\hline $\begin{array}{l}\text { Groups } \\
\text { Parameters }\end{array}$ & Control & Sham & $\begin{array}{c}\text { AC } \\
(100 \mathrm{mg} / \mathrm{kg})\end{array}$ & $\begin{array}{c}\text { AC } \\
(200 \mathrm{mg} / \mathrm{kg})\end{array}$ & $\begin{array}{c}\text { AC } \\
(300 \mathrm{mg} / \mathrm{kg})\end{array}$ \\
\hline Cholesterol & $69.8 \pm 2.9$ & $\begin{array}{c}86.57 \pm 1.9 \\
*\end{array}$ & $\begin{array}{c}66.33 \pm 3.3 \\
\beta\end{array}$ & $70.83 \pm 7.24$ & $76.16 \pm 5.2$ \\
\hline $\mathrm{LH}$ & $1.07 \pm 0.2$ & $0.71 \pm 0.03$ & $\begin{array}{c}1.40 \pm 0.3 \\
\alpha \beta\end{array}$ & $\begin{array}{c}1.54 \pm 0.2 \\
\gamma \beta\end{array}$ & $0.85 \pm 0.03$ \\
\hline FSH & $2.8 \pm 0.4$ & $\begin{array}{c}1.03 \pm 0.07 \\
*\end{array}$ & $1.64 \pm 0.45$ & $\begin{array}{c}3.34 \pm 0.5 \\
\pi \beta \gamma\end{array}$ & $1.60 \pm 0.2$ \\
\hline Testosterone & $6.9 \pm 2.7$ & $5.94 \pm 1.8$ & $\begin{array}{c}1.65 \pm 0.3 \\
\beta\end{array}$ & $\begin{array}{c}0.55 \pm 0.1 \\
\beta\end{array}$ & $2.67 \pm 0.6$ \\
\hline Prolactin & $3.45 \pm 0.25$ & $5.16 \pm 0.55$ & $6.49 \pm 0.7$ & $6.28 \pm 1.4$ & $\begin{array}{c}9.84 \pm 1.8 \\
\alpha \gamma \beta\end{array}$ \\
\hline Cortisol & $20.2 \pm 0.8$ & $20.77 \pm 4.8$ & $\begin{array}{c}10.27 \pm 0.2 \\
\beta\end{array}$ & $\begin{array}{c}10.33 \pm 1.40 \\
\beta\end{array}$ & $\begin{array}{c}27.15 \pm 2.4 \\
\alpha \gamma \beta\end{array}$ \\
\hline \multicolumn{6}{|c|}{$\begin{array}{l}\text { * marks a significant change compared with the control group, } \\
\beta \text { represents a significant change compared with the sham group. } \\
\alpha \text { represents a significant change between the minimum and maximum doses of Alhagi Camelorum extract. } \\
\gamma \text { indicates significant change between average and maximum doses of Alhagi Camelorum extract. } \\
\pi \text { represents a significant change in minimum and average doses of Alhagi Camelorum extract. }\end{array}$} \\
\hline
\end{tabular}

icant difference was observed between the average and minimum groups.

Cortisol levels in the sham group did not show any significant changes as compared to the control group, but in the groups receiving minimum and average doses of the extract it was significantly lower than in the sham group. Cortisol levels in the group receiving the highest dose of the extract compared to sham group as well as the group receiving the least and average doses of the extract showed a significant increase $(\mathrm{P}=0.000)$.

\section{Discussion}

Test results showed that by increasing the amount of cholesterol in the sham group, FSH levels decreased. The administration of the extract to hypercholesterolaemic rats increased gonadotropin and prolactin levels and decreased the level of testosterone. The level of cortisol in the group receiving the highest dose of the extract reduced, but it decreased in groups with the minimum and average doses.

Nowadays, with the prevalence of obesity, it has become an important issue that how fertility in men is affected by obesity and fat. In this study, the relationship between increased cholesterol level and functions of pituitarygonadal axis in the sham group was measured. It is important because if obesity is the cause of male infertility, it can be treated. Obesity affects fertility in men by various mechanisms, among which we can refer to changes in gonadotropin secretion from the pituitary gland, changes in sex hormone-binding globulins, decreased libido, sperm DNA damage, and so on. Another important effect of obesity on fertility is the reduced testicular activity. In adipose tissue ten percent of testosterone which is male hormone turns into estradiol which is a female hormone.

Increased estradiol makes the breasts grow larger in men and obese men typically have larger breasts (24-22). However, studies in this field are controversial. For example, Pauli et al. (25) and Relvany et al. (26) showed that weight gain does not affect the fertility of the semen quality parameters while Wagner et al. (2010) and Paasch et al. (2010) stated that increased BMI has a negative impact on sperm quality and fertility indices (27-28), which is consistent with the results of the present study as it was seen that in the control group by increasing cholesterol, the level of FSH decreased. On the effect of the extract on pituitary - gonadal axis activities it can be said that on one hand, the extract reduced cholesterol, testosterone and on the other, it increased prolactin and gonadotropin levels. One of the most important ways to adjust LH and FSH 
gonadotropin levels is through negative feedback effect of testosterone. That is when the level of this hormone increases, gonadotropin levels decrease and vice versa (29). In this study it seems reasonable that by increasing testosterone gonadotropins decreased.

Leydig cells are the main source of testosterone. Leydig cells have receptors for prolactin that at normal levels increases testosterone. This suggests a collaboration between PRL and LH and testosterone. However, high levels of prolactin, reduce testosterone (1). The results of this study showed that the Alhagi camelorum extract reduced cholesterol and testosterone, but it increased gonadotropins and prolactin.

LHRH hormone can be another possibility for the reduction of testosterone level. This hormone increases LH and FSH hormones and at the same time inhibits testicular testosterone synthesis and secretion by reducing $\mathrm{LH}$ receptors $(30,31)$. Therefore, in this study, despite the increase in FSH and LH, lower testosterone seems to be reasonable and the results of this study is consistent with previous studies on effect of blue plate extract (Centella asiatica) on spermatogenesis, as studies show the chemical compounds in both plants are similar (32).

Testosterone is one of the hormones needed for spermatogenesis. Lower level of this hormone could possibly reduce the number of spermatogonial and spermatocytes cells. These cells produce growth factors such as activin and in the presence of calcium ions, cause karyokinesis, cytokinesis and sperm differentiation (33-34).

Studies on Alhagi camelorum plant indicates that on one hand this herb may inhibit calcium channels and on the other active components of the plant including flavonoids have contraceptives and anti-androgenic effects on reproductive system $(12,13)$.

Alkaloids are key ingredients in this plant. Alkaloids by reducing androgens lead to atrophy of epithelial cells and subsequently prohibits androgenic effects on tissues and thus cancer is treated $(4,35)$. Alkaloids also easily cross the cell membrane and thus they destroy the cytoskeleton, help a variety of free radicals to release and ultimately cause detrimental changes in cellular structure which in turn causes higher activity of white blood cells (anti-inflammatory), while some studies suggest that they have also antioxidant effects $(35,36-38)$

In addition, alkaloids inhibit cholesterol synthesis (39). Similar studies on Berberis indicates that the alkaloid compounds in this plant such as berberine and berbamine can be effective in the prevention of coronary artery diseases and could possibly reduce total cholesterol levels. Blocking calcium channel is the major effect of berbamine. Berberine increases the production of a receptor in the liver that binds with cholesterol and facilitates its disposal $(20,21)$. Since cholesterol is the precursor of steroid hormones, the extract probably lowers testosterone by reducing cholesterol.

Bashtiny et al. in a study on feeding animals with Alhagi camelorum showed that it increased their milk production. This is consistent with our findings as increased prolactin level is one of the most important factors for increasing milk (40). Injecting prolactin and hydrocortisone acetate lactogenic hormone in a variety of lactating mammals induces the synthesis and accumulation of beta-carotene in the mammary glands of rats. Research shows that glucocorticoids can be effective only when they are accompanied by prolactin. Studies also showed that milk secretion is the result of several hormones, and herbal extracts directly stimulate endogenous prolactin secretion. As a result, it works effectively on mammary glands. For example, pectin compounds in plants are capable of stimulating the secretion of prolactin, growth hormone, LH and endorphins from rat pituitary. Most active fractions of the plant that cause prolactin secretion are made of polysaccharides because they have higher amounts of pectin. In addition, there are other compounds involved including prolactin, cortisol and growth hormones (41).

\section{Conclusion}

Based on the findings in this study, Alhagi camelorum extract at the average and minimum doses decreases cortisol, cholesterol, testosterone and increases gonadotropins. So it can cause reproductive disorders in male rats. The extract in the maximum dose can increase cortisol and prolactin. Since these two hormones work together in milk production, this plant can act as a milk booster.

\section{ACKNOWLEDGEMENT}

The authors wish to thank the Deputy of Research of Eghlid Payame Noor University for their kind help and support.

\section{REFERENCES}

1. Fritz MA, Speroff L. Clinical gynecologic endocrinology and infertility. $8^{\text {th }}$ ed. Philadelphia: Lippincott Williams EWilkins. 2010; $157-180$

2. Hajishafiha M, Garehagaji $R$, Salemi $S$, et al. Survey of Association among BMI with Semen Factors and Sex Hormones in Men. Medical Journal of Mashad University of Medical Sciences. 2012; 55:102-109 [in Persian].

3. Murray R, Bender D, Botham KM, editors. Harpers illustrated biochemistry. 29th ed. New York: McGraw Hill. 2012; 415-456.

4. Zarei A, Changizi-Ashtiyani S, Rezaei A, et al. The Effect of Chelidonium majus Extract on the Lipid Profile and Activity of Pituitary-Gonadal Axis in Hypercholesterolemic Rats. Zahedan J Res Med Sci 2014; 16:18-22.

5. Marashdah MS, Farraj AI. Pharmacological activity of 2\% aqueous acetic acid extract of Alhagi maurorum roots. Journal of Saudi Chemical Society. 2010; 14:247-250.

6. Awaad Amani AS. Maitland DJ,. Soliman GA. Antiulcerogenic Activity of Alhagi maurorum Pharmaceutical Biology, 2006; 44:292-296.

7. Samejo MQ. Memon S, Bhanger MI, Khan KM. Chemical composition of essential of essential oils from Alhagi maurorum. Chemistry of Natural Compounds. 2012; 48:898-900.

8. Behari M, Gupt SC The isolation and biogenesis of 24-alkylsterols in Alhagi pseudoalhagi. Acta Cienc Indica Chem. 1980; 6:207-208.

9. Al-Yahya MA, Mossa JS, Al-Badr AA, et al. Phytochemical and biological studies on Saudi medicinal plants Part 12. A study on Saudi plants of family Leguminosae. Int J Crud Drug Res. 1987; 25:65-71. 
10. Singh VP, Bineeta Y, Pandey VB. Flavanone glycosides from Alhagi pseudalhagi. Phytochemistry 1999; 51:587-590.

11. Shaker E, Mahmoud H, Mnaa S. Anti-inflammatory and antiulcer activity of the extract from Alhagi maurorum (camelthorn). Food Chem Toxicol. 2010; 48:2785-90.

12. Bhargava SK. Anti-fertility effects of the flavonoids of Vitex negundo L. seeds in dogs. Plant Med. 198; 20:188-197.

13. Bhargava SK. Antiandrogenic effects of the flavonoid - rich fraction of Vitex negundo seeds: a histological and biochemical study in dogs. J Ethnopharmacol. 1989; 27:327-39.

14. Vuong QV, Hirun S, Phillips PA, et al. Fruit-derived phenolic compounds and pancreatic cancer: Perspectives from Australian native fruits. J Ethnopharmacol. 2014; 152:227-242.

15. Pérez-Carreón JI1, Cruz-Jiménez G, Licea-Vega JA, et al. Genotoxic and anti-genotoxic properties of Calendula officinalis extracts in rat liver cell cultures treated with diethylnitrosamine. Toxicol In Vitro. 2002; 16:253-8.

16. Pyo YH, Lee TC, Logendra L, Rosen RT. Antioxidant activity and phenolic compounds of Swiss chard (Beta valgaris subspecies cycla) extracts. Food chemistry. 2004; 85:19-26.

17. Mitra SK, Venkataranganna MV, Sundaram R, Gopumad havan S. Protective effect of HD-O3, a herbal formulatin, against various hepatotoxic agents in rats. J Ethnopharmacology. 1998; 63:181-186.

18. Sun F, Hayami S, Ogiri Y, et al. Evaluation of oxidative stress based on lipid hydroperoxide, vitamin $C$ and vitamin $E$ during apoptosis and necrosis caused by thioacetamide in rat liver. Biochim Biophys Acta. 2000; 1500:181-5.

19. Yang CS, Landau JM, Huang MT, Newmark HL. Inhibition of carcinogenesis by dietary polyphenolic compounds. Annu Rev Nutr. 2001; 21:381-406.

20. Changizi Ashtiyani S, Zarei A, Taheri S, et al. The effects of Portulaca oleracea alcoholic extract on induced hypercholesteroleomia in rats. Zahedan J Res Med Sci. 2012; 15:33-38.

21. Changizi Ashtiyani S, Zarei A, Taheri S, et al.. A Comparative Study of Hypolipidemic Activities of the Extracts of Melissa officinalis and Berberis vulgaris in Rats. Journal of Medicinal Plants. 2013; 12:38-47.

22. Haghighi S, Yaghmaei P, Hashemi F, et al. The association between serum chemerin concentration and polycystic ovarian syndrome Tehran University Medical Journal. 2012; 70:320-324. [in Persian].

23. Sarvari A, Naderi MM, Heidari $M$, et al. Effect of Environmental Risk Factors on Human Fertility. Medical Journal of Reproduction \& Infertility. 2011; 11: 211-226 [in Persian].

24. Chobine H, Sedighe Gilani MA, Hassanzadeh GH, et al. Assessment of Socio-Demographic Characteristics of Infertile Men Who Referred to Shariati Hospital, Tehran, Iran. IJOGI. 2013; 16:6-12.

25. Pauli EM, Legro RS, Demers LM, et al. Diminished paternity and gonadal function with increasing obesity in men, Fertil Steril. 2008; 90:346-51.

26. Relwani $R$, Berger D, Santoro N, et al. Semen parameters are unrelated to BMI but vary with SSRI use and prior urological surgery. Reprod Sci. 2011; 18:391-7.

27. Wegner C, Clifford AL, Jilbert PM, et al. Abnormally high body mass index and tobacco use are associated with poor sperm quality as revealed by reduced sperm binding to hyaluronan-coated slides. Fertil Steril. 2010; 93:332-4.
28. Paasch U, Grunewald S, Kratzsch J, Glander H. Obesity and age affect male fertility potential. Fertil Steril. 2010; 94:2898-901.

29. Hall JE. Guyton and Hall textbook of medical physiology. 12th ed. Philadelphia: WB. Saunders, 2010; 881-976.

30. Modaresi M, Messripour M, Toghyani M, Rajaii RA. Effect of hydroalcoholic extract of Cinnamon zeylanicum (Bark) on mice pituitary-testis axis. Gorgan Univ Med Sci J. 2010; 12:15-19.

31. Melmed S, Polonsky KS. Williams textbook of endocrinology. 12th Ed. Philadelphia: WB. Saunders; 2012; 581-778.

32. Heidari M, Jamshedi AH, Akhondzadeh Sh, et al. Evaluating the effects of Centella asiatica on spermatogenesis in rats. Medical Journal of Reproduction \& Infertility. 2006; 7:367-374.

33. Ahmadi A, Nasiri Nejad, F, Parivar K. Effect of Aqueous Extract of the Aerial Part of the Ruta Graveolens on the Spermatogenesis of Immature Balb/C Mice. RJMS. 2007; 14:13-20.

34. Grootegoed JA, Siep M, Baarends WM. Molecular and cellular mechanisms in spermatogenesis. Baillieres Pract Res Clin Endocrinol Metab. 2000; 14:331-43.

35. Rahimi-Movaghar A, Khastoo G, Fekri M, Akhondzadeh S. Treatment of addiction by medicinal herbs sellers in Tehran. Hakim Res J. 2008; 11:11-19.

36. Qiu L, Zhao F, Liu H, et al. Two new megastigmane glycosides, physanosides $A$ and B, from Physalis alkekengi L. var. franchetii, and their effect on NO release in macrophages. Chem Biodivers. 2008; 5:758-63.

37. Zarei A, Shariati M, Shekar-Forosh S, et al. The effect of Physalis alkekengi extract on the physiologic function of organ tissues] Persian. Arak Univ. J 2012; 15:94-104 [in Persian].

38. Chand N, Durrani FR, Qureshi MS, Durrani Z. Role of Berberis lycium in reducing serum cholesterol in broilers. Asian-Aust J Anim Sci. 2007; 20:563-568.

39. Zarei A, Changizi Ashtiyani S, Rasekh F, Mohammadi A. The effect of Physalis Alkekengi extracts on lipids concentrations in rats. Arak university of Medical Journal. 2011; 14:36-42 [in Persian].

40. Bashtini J, Fazaeli H, Mirhadi A, Malekkhahi M. Effect of feeding Alhaji browse to lactating ewes on milk yield and performance of lambs. Animal science researches. 2013; 23:39-48.

41. Sepehri H. La synthese de Beta casein par lacide pectique obtenu des extraits des plantes lactogenes. Journal of science, University of Tehran. 1991; 20:61-72.

\section{Correspondence}

Ali Zarei, PhD

Young Researchers Club, Abadeh Branch, Islamic Azad University, Abadeh, Iran

Saeed Changizi Ashtiyani, PhD (Correspondig Author)

dr.ashtiyani@arakmu.ac.ir

Department of Physiology, Arak University of Medical Sciences, Arak, Iran

Gholam Hassan Vaezi, PhD

Department of biology, Islamic Azad University, Damghan Branch, Semnan, Iran 